\title{
ABSOLUTELY MINIMUM ATTAINING CLOSED OPERATORS
}

\author{
S. H. KULKARNI ${ }^{1}$ AND G. RAMESH ${ }^{2} *$
}

\begin{abstract}
We define and discuss properties of the class of unbounded operators which attain minimum modulus. We establish a relationship between this class and the class of norm attaining bounded operators and compare the properties of both. Also we define absolutely minimum attaining operators (possibly unbounded) and characterize injective absolutely minimum attaining operators as those with compact generalized inverse. We give several consequences, one of those is that every such operator has a non trivial hyper invariant subspace.
\end{abstract}

\section{InTRODUCTION}

The class of norm attaining operators on Banach spaces is well studied by several authors in the literature. It is known that the class of norm attaining operators is dense in the space of all bounded linear operators on a Hilbert space with respect to the operator norm [18, Theorem 1]. For more details on norm attaining operators on Banach spaces, we refer to [19, 2] and [14] and references therein.

Every compact operator is norm attaining. In fact, restricted to any non zero closed subspace of a Hilbert space, it remains as compact and hence norm attaining. Motivated by this observation, Carvajal and Neves [12] introduced a class of operators, called the absolutely norm attaining operators. Characterization of such operators on separable Hilbert space, in a particular case is given in [21] and a complete characterization on arbitrary Hilbert space is discussed in [28]. Many properties of these operators resemble the properties of compact operators.

It is a natural question to ask what happens if the norm is replaced by the minimum modulus. This leads to the definition of minimum attaining operators. Analogously, we can define absolutely minimum attaining operators. In a recent paper Carvajal and Neves [13], studied bounded operators between two different Hilbert spaces having such property. The structure of positive absolutely minimum attaining operators is described in [16]. This concept is also applicable to linear operators, that are not bounded.

In this article, we introduce the minimum attaining property for densely defined closed operators (possibly not bounded). We prove several characterizations of such operators. We also prove the dual relation between the norm attaining

Date: April 10, 2019.

${ }^{*}$ Corresponding author.

2000 Mathematics Subject Classification. 47A75, 47A05, 47A10, 47A15 .

Key words and phrases. closed operator, minimum modulus, absolutely minimum attaining operator, invariant subspace, Lomonosov theorem, generalized inverse. 
bounded operators and the minimum attaining closed operators. Finally, we introduce the absolutely minimum attaining operators and prove a representation theorem for the injective absolutely minimum attaining operators. Furthermore, we observe that this class is exactly the same as the class of densely defined closed operators whose generalized inverse is compact. Finally, we show that these operators possess a non trivial hyperinvariant subspace.

We organize the article as follows: In the second section we provide basic results which will be used throughout the article. In the third section, we define minimum attaining property for densely defined closed operators and prove several characterizations. Some of the results in this section generalize the existing results of bounded operators and some of them are new. In the fourth section, we define absolutely minimum attaining operators and show that all such operators have a closed range. In particular, we show that an injective densely defined closed operator is absolutely minimum attaining if and only if its Moore-Penrose inverse is compact. Using this result, we deduce several consequences. One of the important consequences is that every such operator has a non trivial hyper invariant subspace.

\section{Preliminaries}

In this section we introduce some basic notations, definitions and results that are needed to prove our main results.

Throughout the article we consider infinite dimensional complex Hilbert spaces which will be denoted by $H, H_{1}, H_{2}$ etc. The inner product and the induced norm are denoted by $\langle\cdot\rangle$ and $\|$.$\| , respectively. Let T$ be a linear operator with domain $D(T)$, a subspace of $H_{1}$ and taking values in $H_{2}$. If $D(T)$ is dense in $H_{1}$, then $T$ is called a densely defined operator. The graph $G(T)$ of $T$ is defined by $G(T):=\{(T x, x): x \in D(T)\} \subseteq H_{1} \times H_{2}$. If $G(T)$ is closed, then $T$ is called a closed operator. Equivalently, $T$ is closed if and only if $\left(x_{n}\right)$ is a sequence in $D(T)$ such that $x_{n} \rightarrow x \in H_{1}$ and $T x_{n} \rightarrow y \in H_{2}$, then $x \in D(T)$ and $T x=y$.

By the closed graph Theorem [7], an everywhere defined closed operator is bounded. Hence the domain of an unbounded closed operator is a proper subspace of a Hilbert space.

The space of all bounded operators between $H_{1}$ and $H_{2}$ is denoted by $\mathcal{B}\left(H_{1}, H_{2}\right)$ and the class of all closed operators between $H_{1}$ and $H_{2}$ is denoted by $\mathcal{C}\left(H_{1}, H_{2}\right)$. We write $\mathcal{B}(H, H)=\mathcal{B}(H)$ and $\mathcal{C}(H, H)=\mathcal{C}(H)$.

If $T \in \mathcal{C}\left(H_{1}, H_{2}\right)$, then the null space and the range space of $T$ are denoted by $N(T)$ and $R(T)$ respectively and the space $C(T):=D(T) \cap N(T)^{\perp}$ is called the carrier of $T$. In fact, $D(T)=N(T) \oplus^{\perp} C(T)$ [6, page 340].

For a densely defined operator, there exists a unique linear operator (in fact, a closed operator) $T^{*}: D\left(T^{*}\right) \rightarrow H_{1}$, with

$$
D\left(T^{*}\right):=\left\{y \in H_{2}: x \rightarrow\langle T x, y\rangle \text { for all } x \in D(T) \text { is continuous }\right\} \subseteq H_{2}
$$

satisfying $\langle T x, y\rangle=\left\langle x, T^{*} y\right\rangle$ for all $x \in D(T)$ and $y \in D\left(T^{*}\right)$. it is to be noted that $T^{*}$ exists if and only if $T$ is densely defined. 
If $S$ and $T$ are closed operators with the property that $D(T) \subseteq D(S)$ and $T x=S x$ for all $x \in D(T)$, then $S$ is called the restriction of $T$ and $T$ is called an extension of $S$. Furthermore, $S=T$ if and only if $S \subseteq T$ and $T \subseteq S$.

If $S \in \mathcal{B}(H)$ and $T \in \mathcal{C}(H)$ is densely defined, the we say $S$ and $T$ are commuting if $S T \subseteq T S$. That is, $D(S T) \subseteq D(T S)$ and $S T x=T S x$ for all $x \in D(S T)$.

A densely defined operator $T \in \mathcal{C}(H)$ is said to be normal if $T^{*} T=T T^{*}$, self-adjoint if $T=T^{*}$ and positive if $\langle T x, x\rangle \geq 0$ for all $x \in D(T)$.

If $T$ is positive, then there exists a unique positive operator $S$ such that $T=S^{2}$. The operator $S$ is called the square root of $T$ and it is denoted by $S=T^{\frac{1}{2}}$.

If $T \in \mathcal{C}\left(H_{1}, H_{2}\right)$ is densely defined, then the operator $|T|:=\left(T^{*} T\right)^{\frac{1}{2}}$ is called the modulus of $T$. There exists a unique partial isometry $V: H_{1} \rightarrow H_{2}$ with initial space $\overline{R\left(T^{*}\right)}$ and range $\overline{R(T)}$ such that $T=V|T|$.

It can be verified that $D(|T|)=D(T)$ and $N(|T|)=N(T)$ and $\overline{R(|T|)}=\overline{R\left(T^{*}\right)}$.

Let $T \in \mathcal{C}(H)$ be densely defined. The resolvent of $T$ is defined by

$$
\rho(T):=\left\{\lambda \in \mathbb{C}: T-\lambda I: D(T) \rightarrow H \text { is invertible and }(T-\lambda I)^{-1} \in \mathcal{B}(H)\right\}
$$

and

$$
\begin{aligned}
\sigma(T) & :=\mathbb{C} \backslash \rho(T) \\
\sigma_{p}(T) & :=\{\lambda \in \mathbb{C}: T-\lambda I: D(T) \rightarrow H \text { is not one-to-one }\},
\end{aligned}
$$

are called the spectrum and the point spectrum of $T$, respectively.

Let $T \in \mathcal{C}\left(H_{1}, H_{2}\right)$ be densely defined. A subspace $D$ of $D(T)$ is called a core for $T$ if for any $x \in D(T)$, there exists a sequence $\left(x_{n}\right) \subset D$ such that $\lim _{n \rightarrow \infty} x_{n}=x$ and $\lim _{n \rightarrow \infty} T x_{n}=T x$. In other words, $D$ is dense in the graph norm, which is defined by $\||x\|\mid=\| x\|+\| T x \|$ for all $x \in D(T)$. It is a well known fact that $D\left(T^{*} T\right)$ is a core for $T$ (see [27, Proposition 3.18, page 47] for details).

If $M$ is a closed subspace of a Hilbert space $H$, then $P_{M}$ denotes the orthogonal projection $P_{M}: H \rightarrow H$ with range $M$, and $S_{M}:=\{x \in M:\|x\|=1\}$ is the unit sphere of $M$.

We refer $[1,4,5,7,20,27]$ for the above basics of unbounded operators.

Here we recall definition and properties of the Moore-Penrose inverse (or generalized inverse) of a densely defined closed operator that we need for our purpose. We refer [6] for more details on this topic.

Let $T \in \mathcal{C}\left(H_{1}, H_{2}\right)$ be densely defined. Then there exists a unique densely defined operator $T^{\dagger} \in \mathcal{C}\left(H_{2}, H_{1}\right)$ with domain $D\left(T^{\dagger}\right)=R(T) \oplus^{\perp} R(T)^{\perp}$ and has the following properties:

(1) $T T^{\dagger} y=P_{\overline{R(T)}} y$, for all $y \in D\left(T^{\dagger}\right)$

(2) $T^{\dagger} T x=P_{N(T) \perp} x$, for all $x \in D(T)$

(3) $N\left(T^{\dagger}\right)=R(T)^{\perp}$.

This unique operator $T^{\dagger}$ is called the Moore-Penrose inverse of $T$.

The following property of $T^{\dagger}$ is also well known.

For every $y \in D\left(T^{\dagger}\right)$, let

$$
L(y):=\{x \in D(T):\|T x-y\| \leq\|T u-y\| \text { for all } u \in D(T)\} .
$$


Here any $u \in L(y)$ is called a least square solution of the operator equation $T x=y$. The vector $x=T^{\dagger} y \in L(y),\left\|T^{\dagger} y\right\| \leq\|x\|$ for all $x \in L(y)$ and it is called the least square solution of minimal norm. A different treatment of $T^{\dagger}$ is given in [6, Pages 314, 318-320], where it is called "the Maximal Tseng generalized Inverse".

Here we recall some properties of $T^{\dagger}$ that we will be using very frequently.

Theorem 2.1. [6, Page 320] Let $T \in \mathcal{C}\left(H_{1}, H_{2}\right)$ be densely defined. Then

(1) $D\left(T^{\dagger}\right)=R(T) \oplus^{\perp} R(T)^{\perp}, N\left(T^{\dagger}\right)=R(T)^{\perp}=N\left(T^{*}\right)$

(2) $R\left(T^{\dagger}\right)=C(T)$

(3) $T^{\dagger}$ is densely defined and $T^{\dagger} \in \mathcal{C}\left(H_{2}, H_{1}\right)$

(4) $T^{\dagger}$ is continuous if and only $R(T)$ is closed

(5) $T^{\dagger \dagger}=T$

(6) $T^{* \dagger}=T^{\dagger *}$

(7) $N\left(T^{* \dagger}\right)=N(T)$

(8) $T^{*} T$ and $T^{\dagger} T^{* \dagger}$ are positive and $\left(T^{*} T\right)^{\dagger}=T^{\dagger} T^{* \dagger}$

(9) $T T^{*}$ and $T^{* \dagger} T^{\dagger}$ are positive and $\left(T T^{*}\right)^{\dagger}=T^{* \dagger} T^{\dagger}$.

\section{Minimum attaining Operators}

In this section first we discuss some important properties of minimum attaining operators. These operators for the bounded case was discussed in [13] and the unbounded case in [25]. It is proved that this class is dense in the class of densely defined closed operators with respect to the gap topology.

Definition 3.1. [6, 5] Let $T \in \mathcal{C}\left(H_{1}, H_{2}\right)$ be densely defined. Then

$$
\begin{aligned}
m(T) & :=\inf \left\{\|T x\|: x \in S_{D(T)}\right\} \\
\gamma(T) & :=\inf \left\{\|T x\|: x \in S_{C(T)}\right\},
\end{aligned}
$$

are called the minimum modulus and the reduced minimum modulus of $T$, respectively. The operator $T$ is said to be bounded below if and only if $m(T)>0$.

Remark 3.2. If $T \in \mathcal{C}\left(H_{1}, H_{2}\right)$ is densely defined, then

(a) By definition, we have $m(T) \leq \gamma(T)$. More over, if $T$ is one-to-one, $m(T)=\gamma(T)$ since $D(T)=C(T)$

(b) $m(T)>0$ if and only if $R(T)$ is closed and $T$ is one-to-one

(c) Since $D(T)=D(|T|)$ and $\|T x\|=\||T| x\|$ for all $x \in D(T)$, we can conclude that $m(T)=m(|T|)$ and $\gamma(T)=\gamma(|T|)$.

Remark 3.3. If $T \in \mathcal{C}(H)$ is densely defined and $R(T)$ is closed, then $\gamma(T)=$ $\frac{1}{\left\|T^{\dagger}\right\|}$.

Recall that $T \in \mathcal{B}\left(H_{1}, H_{2}\right)$ is said to be norm attaining if there exists $x_{0} \in S_{H_{1}}$ such that $\left\|T x_{0}\right\|=\|T\|$. We denote the class of all norm attaining operators between $H_{1}$ and $H_{2}$ by $\mathcal{N}\left(H_{1}, H_{2}\right)$. In case $H_{1}=H_{2}=H$, we denote this by $\mathcal{N}(H)$. In a similar way, we can define operators that attain minimum modulus. The class of bounded operators that attain minimum modulus is defined and several characterizations are proved in [12]. Here we discuss the same for unbounded operators. 
Definition 3.4. Let $T \in \mathcal{C}\left(H_{1}, H_{2}\right)$ be densely defined. If there exists $x_{0} \in S_{D(T)}$ such that $\left\|T x_{0}\right\|=m(T)$, then we call $T$ to be minimum attaining.

We write

$\mathcal{M}_{c}\left(H_{1}, H_{2}\right)=\left\{T \in \mathcal{C}\left(H_{1}, H_{2}\right): T\right.$ is densely defined and minimum attaining $\}$ and $\mathcal{M}_{c}(H, H)=\mathcal{M}_{c}(H)$.

Remark 3.5. Let $T \in \mathcal{C}\left(H_{1}, H_{2}\right)$ be densely defined.

(1) If $T$ is not one-to-one, then $m(T)=0$ and there exists a $x_{0} \in S_{N(T)}$ such that $T x_{0}=0$. Hence $T \in \mathcal{M}_{c}\left(H_{1}, H_{2}\right)$.

(2) If $T$ is one-to-one and $R(T)$ is not closed, then $m(T)=0$. But there does not exists $x_{0} \in D(T)$ such that $\left\|T x_{0}\right\|=0$, since $T$ is one-to-one. Thus $T \notin \mathcal{M}_{c}\left(H_{1}, H_{2}\right)$.

From the above two observations it is apparent that the injectivity of the operator plays an important role in the minimum attaining property.

First, we establish some results related to the minimum modulus of a densely defined closed operator, which are useful in discussing the minimum attaining property.

Proposition 3.6. Let $T \in \mathcal{C}(H)$ be densely defined and normal. Then

(1) $m(T)=d(0, \sigma(T))$

(2) $m\left(T^{n}\right)=m(T)^{n}$.

Proof. If $m(T)=0$, then $T$ is not invertible, so $0 \in \sigma(T)$ and $d(0, \sigma(T))=0$. If $m(T)>0$, then $T^{-1} \in \mathcal{B}(H)$. In this case, $m(T)=\gamma(T)=\frac{1}{\left\|T^{-1}\right\|}$. Therefore,

$$
\begin{aligned}
\frac{1}{\left\|T^{-1}\right\|} & =\frac{1}{\sup \left\{\mu: \mu \in \sigma\left(T^{-1}\right)\right\}} \\
& =\frac{1}{\sup \left\{\frac{1}{\lambda}: \lambda \in \sigma(T)\right\}} \\
& =\inf \{\lambda: \lambda \in \sigma(T)\} \\
& =d(0, \sigma(T)) .
\end{aligned}
$$

Proof of (2): It is easy to verify that $T^{n}$ is normal. Hence, by (1) and the spectral mapping theorem we can conclude that

$$
\begin{aligned}
m\left(T^{n}\right) & =\inf \left\{|\mu|: \mu \in \sigma\left(T^{n}\right)\right\} \\
& =\inf \left\{\left|\lambda^{n}\right|: \lambda \in \sigma(T)\right\} \\
& =\inf \left\{|\lambda|^{n}: \lambda \in \sigma(T)\right\} \\
& =m(T)^{n} .
\end{aligned}
$$

Corollary 3.7. If $T \in \mathcal{C}\left(H_{1}, H_{2}\right)$ is densely defined, then

(1) $m(T)=d(0, \sigma(|T|))$

(2) $m\left(T^{*} T\right)=m(T)^{2}$.

Proof. We have $m(T)=m(|T|)=d(0, \sigma(|T|))$, by (1) of Proposition 3.6. Also, $m\left(T^{*} T\right)=m\left(|T|^{2}\right)=d\left(0, \sigma\left(|T|^{2}\right)\right)=d(0, \sigma(|T|))^{2}=m(|T|)^{2}=m(T)^{2}$. Here we have used both (1) and (2) of Proposition 3.6 to get the conclusion. 
Proposition 3.8. Let $T \in \mathcal{C}(H)$ be densely defined and positive. Then

$$
m(T)=\inf \left\{\langle T x, x\rangle: x \in S_{D(T)}\right\}=m_{T} .
$$

Proof. First note that $D(T) \subseteq D\left(T^{\frac{1}{2}}\right)$. Next,

$$
\begin{aligned}
m_{T}=\inf \left\{\langle T x, x\rangle: x \in S_{D(T)}\right\} & =\inf \left\{\left\langle T^{\frac{1}{2}} x, T^{\frac{1}{2}} x\right\rangle: x \in S_{D(T)}\right\} \\
& \geq \inf \left\{\left\|T^{\frac{1}{2}} x\right\|^{2}: x \in D\left(T^{\frac{1}{2}}\right)\right\} \\
& =m\left(T^{\frac{1}{2}}\right)^{2} .
\end{aligned}
$$

But $m\left(T^{\frac{1}{2}}\right)=\inf \left\{\lambda: \lambda \in \sigma\left(T^{\frac{1}{2}}\right)\right\}$ by Corollary 3.7. As $\sigma(T)=\left\{\lambda^{2}: \lambda \in \sigma\left(T^{\frac{1}{2}}\right)\right\}$, we have that $m\left(T^{\frac{1}{2}}\right)^{2}=m(T)$ and hence $m_{T} \geq m(T)$.

On the other hand, we have

$$
\begin{aligned}
m_{T} & \leq\langle T x, x\rangle \text { for all } x \in S_{D(T)} \\
& =\left\langle T^{\frac{1}{2}} x, T^{\frac{1}{2}} x\right\rangle \text { for all } x \in S_{D(T)} \\
& =\left\|T^{\frac{1}{2}} x\right\|^{2} \text { for all } x \in S_{D(T)} .
\end{aligned}
$$

Next, we claim that the above inequality holds for all $x \in D\left(T^{\frac{1}{2}}\right)$. To this end, let $x \in D\left(T^{\frac{1}{2}}\right)$. Since, $D(T)$ is a core for $T^{\frac{1}{2}}$, there exists a sequence $\left(x_{n}\right) \subset D(T)$ such that $\lim _{n \rightarrow \infty} x_{n} \rightarrow x$ and $\lim _{n \rightarrow \infty} T^{\frac{1}{2}} x_{n}=T^{\frac{1}{2}} x$. Hence $\left\|T^{\frac{1}{2}} x\right\|^{2}=\lim _{n \rightarrow \infty}\left\|T^{\frac{1}{2}} x_{n}\right\|^{2} \geq$ $m_{T}$. As this is true for all $x \in D\left(T^{\frac{1}{2}}\right)$, it follows that $m(T) \geq m_{T}$.

By the above two observations the conclusion follows.

Proposition 3.9. Let $T \in \mathcal{C}\left(H_{1}, H_{2}\right)$ be densely defined and $m(T)=m\left(T^{*}\right)$. Also, assume that $R(T)$ is closed. Then $T \in \mathcal{M}_{c}\left(H_{1}, H_{2}\right)$ if and only if $T^{*} \in$ $\mathcal{M}_{c}\left(H_{2}, H_{1}\right)$.

Proof. Clearly, if $m(T)=m\left(T^{*}\right)=0$, since $R(T)$ closed, both $T$ and $T^{*}$ are not one-to-one. Hence both are minimum attaining. Now assume that $m(T)>0$. It is sufficient to prove one implication, since $T^{* *}=T$ and $m(T)=m\left(T^{*}\right)$. By Proposition 3.13, $T \in \mathcal{M}_{c}\left(H_{1}, H_{2}\right)$ if and only if there exists a $x_{0} \in S_{D(|T|)}$ such that $|T| x_{0}=m(T) x_{0}$. That is $T^{*} T x_{0}=m(T)|T| x_{0}$. Hence

$$
\frac{\left\|T^{*} T x_{0}\right\|}{\left\|T x_{0}\right\|}=m(T) \frac{\left\|T \mid x_{0}\right\|}{\left\|T x_{0}\right\|}=m(T),
$$

proving $T^{*} \in \mathcal{M}_{c}\left(H_{2}, H_{1}\right)$.

Remark 3.10. Let $T \in \mathcal{C}(H)$ be densely defined and normal. Then $D(T)=$ $D\left(T^{*}\right)$ and $\|T x\|=\left\|T^{*} x\right\|$ for all $x \in D(T)$. Hence $T \in \mathcal{M}_{c}(H)$ if and only if $T^{*} \in \mathcal{M}_{c}(H)$. Clearly, in this case $m(T)=m\left(T^{*}\right)$. Note that in this case we don't have to assume that the range of $T$ to be closed.

We recall that if $T \in \mathcal{C}\left(H_{1}, H_{2}\right)$ is densely defined, then the numerical range $W(T)$ of $T$ is defined by $W(T)=\left\{\langle T x, x\rangle: x \in S_{D(T)}\right\}$.

Proposition 3.11. If $T \in \mathcal{C}(H)$ is positive, then the following are equivalent;

(1) $T \in \mathcal{M}_{c}(H)$ 
(2) $m(T) \in \sigma_{p}(T)$

(3) $m(T)$ is an extreme point of $W(T)$.

Proof. Proof of $(1) \Rightarrow(2)$ : Choose $x_{0} \in S_{D(T)}$ such that $\left\|T x_{0}\right\|=m(T)$. Since, $T-m(T) I$ is positive, and by the Cauchy-Scwarz inequality, we get that

$$
m(T) \leq\left\langle T x_{0}, x_{0}\right\rangle \leq\left\|T x_{0}\right\|=m(T),
$$

or $m(T)=\left\langle T x_{0}, x_{0}\right\rangle$. Therefore,

$$
\begin{aligned}
\left\|T x_{0}-m(T) x_{0}\right\|^{2} & =\left\|T x_{0}\right\|^{2}+m(T)^{2}-2 m(T)\left\langle T x_{0}, x_{0}\right\rangle \\
& =2 m(T)^{2}-2 m(T)^{2} \\
& =0 .
\end{aligned}
$$

That is, $T x_{0}=m(T) x_{0}$. Clearly, if $m(T) \in \sigma_{p}(T)$, then $T \in \mathcal{M}_{c}(H)$.

Proof of $(2) \Rightarrow(3)$ : Let $x_{0} \in S_{D(T)}$ be such that $T x_{0}=m(T) x_{0}$. Then $m(T)=\left\langle T x_{0}, x_{0}\right\rangle \in W(T)$. Since, $m(T)=m_{T}$ by Proposition 3.8, the conclusion follows. The other way implication follows by the main theorem of [11].

Using Proposition 3.11, we can prove the following.

Proposition 3.12. Let $T \in \mathcal{C}(H)$ be densely defined and positive. Then $T \in$ $\mathcal{M}_{c}(H)$ if and only if $T^{\frac{1}{2}} \in \mathcal{M}_{c}(H)$.

Proof. If $T^{\frac{1}{2}} \in \mathcal{M}_{c}(H)$, then $m\left(T^{\frac{1}{2}}\right) \in \sigma_{p}\left(T^{\frac{1}{2}}\right)$, which implies that $m(T) \in \sigma_{p}(T)$. By Proposition 3.11, $T \in \mathcal{M}_{c}(H)$.

Conversely, if $T \in \mathcal{M}_{c}(H)$, then $m(T) \in \sigma_{p}(T)$, by Proposition 3.11. If $m(T)=$ 0 , then $m\left(T^{\frac{1}{2}}\right)=0$ and hence $T^{\frac{1}{2}} \in \mathcal{M}_{c}(H)$. Next, assume that $m(T)>0$. Then $m\left(T^{\frac{1}{2}}\right)>0$ and

$$
T-m(T) I=\left(T^{\frac{1}{2}}+m(T)^{\frac{1}{2}} I\right)\left(T^{\frac{1}{2}}-m(T)^{\frac{1}{2}} I\right) .
$$

As $T^{\frac{1}{2}}+m(T)^{\frac{1}{2}} I$ has a bounded inverse, we have that $T^{\frac{1}{2}}-m(T)^{\frac{1}{2}} I$ is not oneto-one. Hence $m\left(T^{\frac{1}{2}}\right) \in \sigma_{p}\left(T^{\frac{1}{2}}\right)$. The conclusion follows by Proposition 3.11.

Theorem 3.13. Let $T \in \mathcal{C}\left(H_{1}, H_{2}\right)$ be densely defined. Then the following statements are equivalent:

(1) $T \in \mathcal{M}_{c}\left(H_{1}, H_{2}\right)$

(2) $|T| \in \mathcal{M}\left(H_{1}\right)$

(3) $T^{*} T \in \mathcal{M}\left(H_{1}\right)$.

Proof. The equivalence of (1) and (2) follows by the observation that $D(T)=$ $D(|T|)$ and $\|T x\|=\||T| x\|$ for all $x \in D(T)$. The equivalence of (2) and (3) follows by the fact that $T^{*} T=|T|^{2}$ and Proposition 3.12.

Example 3.14. Let $D=\left\{\left(x_{n}\right) \in \ell^{2}: \sum_{n=1}^{\infty} n^{2}\left|x_{n}\right|^{2}<\infty\right\}$. Define $T: D \rightarrow \ell^{2}$ by

$$
T\left(\left(x_{1}, x_{2}, x_{3}, \ldots,\right)\right)=\left(\left(0, x_{1}, 2 x_{2}, 3 x_{3}, \ldots\right)\right) \text {, for all }\left(x_{n}\right) \in D .
$$

Clearly, $T$ is densely defined closed operator. Note that $T^{*} T\left(x_{n}\right)=\left(n^{2} x_{n}\right)$ for all $\left(x_{n}\right) \in D\left(T^{*} T\right)$. It can be easily calculated that $\sigma\left(T^{*} T\right)=\sigma_{p}\left(T^{*} T\right)=$ 
$\left\{n^{2}: n \in \mathbb{N}\right\}$. Hence $m\left(T^{*} T\right)=1$ and $T^{*} T \in \mathcal{M}\left(\ell^{2}\right)$. By Theorem 3.13, we can conclude that $T \in \mathcal{M}\left(\ell^{2}\right)$ and by Corollary $3.7 m(T)=1$.

Proposition 3.15. Let $T$ be densely defined and positive. Then $T \in \mathcal{M}_{c}(H)$ if and only if $T^{n} \in \mathcal{M}_{c}(H)$ for each $n \geq 1$.

Proof. Let $T \in \mathcal{M}_{c}(H)$. Then by Proposition 3.11, there exists $x_{0} \in D(T)$ such that $T x_{0}=m(T) x_{0}$. Observe that $x_{0} \in D\left(T^{2}\right)$. This implies that $T^{2} x_{0}=$ $m(T)^{2} x_{0}=m\left(T^{2}\right) x_{0}$, by Proposition 3.6. That is $x_{0} \in D\left(T^{4}\right) \subseteq D\left(T^{3}\right)$. With this, we have $T^{3} x_{0}=m(T)^{3} x_{0}$. By the induction argument we can show that $T^{n} x_{0}=m(T)^{n} x_{0}$. By Proposition 3.11, it follows that $T^{n} \in \mathcal{M}_{c}(H)$.

To prove the converse, assume that $n>1$ and $T^{n} \in \mathcal{M}_{c}(H)$. Choose $x_{0} \in S_{D(T)}$ such that $T^{n} x_{0}=m\left(T^{n}\right) x_{0}$. As $m\left(T^{n}\right)=m(T)^{n}$, if $m\left(T^{n}\right)=0$, then $m(T)=0$. In this case $x_{o} \in N\left(T^{n}\right)$. That is $T^{n-2} x_{0} \in N\left(T^{2}\right)=N(T)$. Hence $x_{0} \in N\left(T^{n-1}\right)$. Proceeding in this we can conclude that $x_{0} \in N(T)$, proving $T \in \mathcal{M}_{c}(H)$.

Next assume that $m(T)>0$. Since $T$ is positive, $T^{-1} \in \mathcal{B}(H)$. Hence $T x_{0}=$ $m(T) x_{0}$ implies that

$$
T^{n-1} x_{0}=T^{-1} T^{n} x_{0}=m(T)^{n} T^{-1} x_{0}=m(T)^{n} \frac{x_{0}}{m(T)}=m(T)^{n-1} x_{0} .
$$

By proceeding in this way, we can conclude that $T x_{0}=m(T) x_{0}$. Hence $T \in$ $\mathcal{M}_{c}(H)$.

Proposition 3.16. Let $T \in \mathcal{M}_{c}\left(H_{1}, H_{2}\right)$ be one-to-one. Then $R(T)$ is closed.

Proof. If $R(T)$ is not closed, then $m(T)=0$. Since $T \in \mathcal{M}_{c}\left(H_{1}, H_{2}\right)$, there exists $x_{0} \in S_{D(T)}$ such that $\left\|T x_{0}\right\|=0$, but contradicts $T$ to be one-to-one. Thus $R(T)$ is closed.

Remark 3.17. The condition one-one ness is not necessary in Proposition 3.16. For example, let $P$ be a bounded orthogonal projection. Then $R(P)$ is closed and it is minimum attaining but not one-to-one.

Corollary 3.18. Let $T \in \mathcal{M}_{c}\left(H_{1}, H_{2}\right)$. Then $T$ is one-to-one if and only $T$ bounded below.

Next, we will establish a relation between the minimum attaining property of the operator and the norm attaining property of its generalized inverse. First we prove a few results needed for this purpose.

Proposition 3.19. Let $T \in \mathcal{C}\left(H_{1}, H_{2}\right)$ be densely defined. Then

(1) $\left|T^{\dagger}\right|=\left|T^{*}\right|^{\dagger}$

(2) $\left|\left(T^{\dagger}\right)^{*}\right|=|T|^{\dagger}$.

Proof. Proof of (1): By definition of $\left|T^{\dagger}\right|$, and by Theorem 2.1,

$$
\left|T^{\dagger}\right|=\left(\left(T^{\dagger}\right)^{*} T^{\dagger}\right)^{\frac{1}{2}}=\left(\left(T T^{*}\right)^{\dagger}\right)^{\frac{1}{2}}=\left(\left(\left|T^{*}\right|^{2}\right)^{\dagger}\right)^{\frac{1}{2}}=\left(\left(\left|T^{*}\right|\right)^{\dagger}\left(\left|T^{*}\right|\right)^{\dagger}\right)^{\frac{1}{2}}=\left|T^{*}\right|^{\dagger} .
$$

The proof of (2) can be obtained by replacing $T$ by $T^{*}$ in (1) and observing that $\left(T^{*}\right)^{\dagger}=\left(T^{*}\right)^{\dagger}$ and $\left(T^{*}\right)^{*}=T$.

Theorem 3.20. Let $T \in \mathcal{C}\left(H_{1}, H_{2}\right)$ be densely defined and one-to-one. Then the following statements are equivalent; 
(1) $T \in \mathcal{M}_{c}\left(H_{1}, H_{2}\right)$

(2) $R(T)$ is closed and $T^{\dagger} \in \mathcal{N}\left(H_{2}, H_{1}\right)$.

Proof. First assume that $T \in \mathcal{M}_{c}\left(H_{1}, H_{2}\right)$. Note that $R(T)$ is closed, by Proposition 3.16. As $T$ is one-to-one, $m(T)>0$. Choose $x_{0} \in D(T)$ such that $|T| x_{0}=m(T) x_{0}$. Hence

$$
m(T)|T|^{-1} x_{0}=|T|^{-1}|T| x_{0}=x_{0} .
$$

So

$$
|T|^{-1} x_{0}=\frac{1}{m(|T|)} x_{0}
$$

By Proposition 3.19, we have $|T|^{-1}=\left|\left(T^{\dagger}\right)^{*}\right|=\left(T^{*}\right)^{\dagger}$. Since $m(|T|)=m(T)=$ $\frac{1}{\left\|T^{\dagger}\right\|}=\frac{1}{\left\|\left|T^{\dagger}\right|\right\|}$, Equation 3.1, takes the form $\left|T^{\dagger}\right| x_{0}=\left\|\left|T^{\dagger}\right|\right\| x_{0}$. Hence the conclusion follows.

To prove the other implication, let $T^{\dagger} \in \mathcal{N}\left(H_{2}, H_{1}\right)$. Then clearly, $R(T)$ is closed. By [12, Proposition 2.5], $S:=\left(T^{\dagger}\right)^{*} \in \mathcal{N}\left(H_{1}, H_{2}\right)$. Hence by Proposition 3.19, we have $|S|=|T|^{\dagger}$. Since $|T|^{\dagger}$ is positive and norm attaining, there exists $x_{0} \in S_{H_{1}}$ such that

$$
|S| x_{0}=\|S\| x_{0}=\left\|T^{\dagger}\right\| x_{0}=\frac{1}{m(T)} x_{0}
$$

Note that $x_{0} \in R\left(|T|^{\dagger}\right)=C(|T|)=C(T) \subseteq N(T)^{\perp}$. Premultiplying Equation 3.2 by $|T|$ and noting that $R(|T|)=N(T)^{\perp}$, we have $|T| x_{0}=m(T) x_{0}$, concluding $|T| \in \mathcal{M}\left(H_{1}\right)$. Hence $T \in \mathcal{M}_{c}\left(H_{1}, H_{2}\right)$ by Theorem 3.13.

Next, we show that minimum attaining property of a closed densely defined operator is related to the minimum attaining property of the corresponding bounded transform. If $T \in \mathcal{C}\left(H_{1}, H_{2}\right)$ is densely defined, then the operator $Z_{T}:=T\left(I+T^{*} T\right)^{-\frac{1}{2}}$ is called the bounded transform of $T$. More over, $T=$ $Z_{T}\left(I-Z_{T}^{*} Z_{T}\right)^{-\frac{1}{2}}$. We refer [27, section 7.3 , page 142$]$ for more details about these operators.

Proposition 3.21. Let $T \in \mathcal{C}\left(H_{1}, H_{2}\right)$ be densely defined. Then

(1) $m\left(Z_{T}\right)=\frac{m(T)}{\sqrt{1+m(T)^{2}}}$

(2) $m(T)^{2}=\frac{m\left(Z_{T}\right)^{2}}{1-m\left(Z_{T}\right)^{2}}$

(3) $T \in \mathcal{M}_{c}\left(H_{1}, H_{2}\right)$ if and only if $Z_{T} \in \mathcal{M}_{c}\left(H_{1}, H_{2}\right)$.

Proof. Proof of (1): We have that

$m\left(Z_{T}\right)^{2}=m\left(Z_{T}^{*} Z_{T}\right)=m\left(I-\left(I+T^{*} T\right)^{-1}\right)=1-\left\|\left(I+T^{*} T\right)^{-1}\right\|=1-\frac{1}{m\left(I+T^{*} T\right)}$, 
by Remark 3.3. Hence

$$
\begin{aligned}
m\left(Z_{T}\right)^{2} & =1-\frac{1}{1+m(T)^{2}} \\
& =\frac{m(T)^{2}}{1+m(T)^{2}} .
\end{aligned}
$$

Proof of (2): Note that $T^{*} T=Z_{T}^{*} Z_{T}\left(I-Z_{T}^{*} Z_{T}\right)^{-1}=\left(I-Z_{T}^{*} Z_{T}\right)^{-1}-I$. Thus $m(T)^{2}=m\left(T^{*} T\right)=m\left(I-Z_{T}^{*} Z_{T}\right)^{-1}-1=\frac{1}{\left\|I-Z_{T}^{*} Z_{T}\right\|}-1=\frac{1}{1-m\left(Z_{T}^{*} Z_{T}\right)}-1$, by Remark 3.3. Hence

$$
m(T)^{2}=\frac{1}{1-m\left(Z_{T}\right)^{2}}-1=\frac{m\left(Z_{T}\right)^{2}}{1-m\left(Z_{T}\right)^{2}} .
$$

Proof of (3): In view of Theorem 3.13, it is enough to prove $T^{*} T \in \mathcal{M}\left(H_{1}\right)$ if and only if $Z_{T}^{*} Z_{T} \in \mathcal{M}\left(H_{1}\right)$. We know by (2) of Proposition 3.11, that $T^{*} T \in$ $\mathcal{M}\left(H_{1}\right)$ if and only if $m(T)^{2} \in \sigma_{p}\left(T^{*} T\right)$. Since, $Z_{T}^{*} Z_{T}=T^{*} T\left(I+T^{*} T\right)^{-1}$ and $m\left(Z_{T}^{*} Z_{T}\right)=\frac{m(T)^{2}}{1+m(T)^{2}}$, it can be verified that $m(T)^{2} \in \sigma_{p}\left(T^{*} T\right)$ if and only if $m\left(Z_{T}^{*} Z_{T}\right) \in \sigma_{p}\left(Z_{T}^{*} Z_{T}\right)$.

\section{Absolutely minimum attaining operators}

In this section, we define absolutely minimum attaining operators and describe the structure of such operators.

Definition 4.1. Let $T \in \mathcal{C}\left(H_{1}, H_{2}\right)$ be densely defined. Then $T$ is called absolutely minimum attaining operator if $\left.T\right|_{M}: D(T) \cap M \rightarrow H_{2}$ is minimum attaining for each non-zero closed subspace $M$ of $H_{1}$. In other words, $T$ is absolutely minimum attaining if there exists $x_{0} \in D\left(\left.T\right|_{M}\right)$ with $\left\|x_{0}\right\|=1$ such that $\left\|T x_{0}\right\|=m\left(\left.T\right|_{M}\right)$.

Note that if $T \in \mathcal{C}\left(H_{1}, H_{2}\right)$ is densely defined and $M$ is a closed subspace of $H$, then the restriction operator $\left.T\right|_{M}: D(T) \cap M \rightarrow H_{2}$ is a closed operator and it is densely defined as $D\left(\left.T\right|_{M}\right)$ is dense in the Hilbert space $\overline{D\left(\left.T\right|_{M}\right)}$.

We denote the set of all absolutely minimum attaining operators between $H_{1}$ and $H_{2}$ by $\mathcal{A M}_{c}\left(H_{1}, H_{2}\right)$ and in case if $H_{1}=H_{2}=H$, this is denoted by $\mathcal{A M}_{c}(H)$. This class of operators was introduced and studied in detail by Carvajal and Neves in [13]. The structure of positive absolutely minimum attaining bounded operators is studied in [16].

Proposition 4.2. Let $T \in \mathcal{A M}_{c}\left(H_{1}, H_{2}\right)$. Then $R(T)$ is closed.

Proof. Since $T \in \mathcal{A M}_{c}\left(H_{1}, H_{2}\right)$, we have $T_{0}=\left.T\right|_{N(T)^{\perp}} \in \mathcal{A M}_{c}\left(N(T)^{\perp}, H_{2}\right)$ and one-to-one. Hence by Proposition 3.16, $R\left(T_{0}\right)$ is closed. It is clear that $R(T)=R\left(T_{0}\right)$.

Remark 4.3. The converse of Proposition 4.2 need not be true. Let $P$ be a bounded orthogonal projection with infinite dimensional null space and infinite dimensional range space. Then $R(P)$ is closed but $P$ is not absolutely minimum attaining by [13, Lemma 3.2]. 
Let $M$ be a closed subspace of $H$ and $T \in \mathcal{C}(H)$ be densely defined. Then $M$ is said to be invariant under $T$, if $T(M \cap D(T)) \subseteq M$.

Let $P:=P_{M}$. If $P(D(T)) \subseteq D(T)$ and $(I-P)(D(T)) \subseteq D(T)$, then

$$
T=\left(\begin{array}{ll}
T_{11} & T_{12} \\
T_{21} & T_{22}
\end{array}\right)
$$

where $T_{i j}=P_{i} T P_{j} \mid M_{j}(i, j=1,2)$. Here $P_{1}=P$ and $P_{2}=I-P$. It is known that $M$ is invariant under $T$ if and only if $T_{21}=0$. Also, $M$ reduces $T$ if and only if $T_{21}=0=T_{12}$.

Remark 4.4. Let $T \in \mathcal{C}\left(H_{1}, H_{2}\right)$ be densely defined. Assume that $M$ reduces $T$ and $T_{1}=\left.T\right|_{M}$ and $T_{2}=\left.T\right|_{M^{\perp}}$. Then the following can be easily verified:

(1) $m(T)=\min \left\{m\left(T_{1}\right), m\left(T_{2}\right)\right\}$

(2) $T \in \mathcal{M}_{c}(H)$ if and only if the operator $T_{j}$ with $m\left(T_{j}\right)=m(T), \quad(j=$ 1 or 2$)$, is minimum attaining.

Lemma 4.5. Let $T \in \mathcal{C}(H)$ be densely defined. If $M$ reduces $T$, then $\left.T^{\dagger}\right|_{M}=$ $\left(\left.T\right|_{M}\right)^{\dagger}$.

Proof. Since $M$ is reducing subspace, we have

$$
T=\left(\begin{array}{cc}
T_{1} & 0 \\
0 & T_{2}
\end{array}\right)
$$

where $T_{1}=\left.T\right|_{M}$ and $T_{2}=\left.T\right|_{M^{\perp}}$. Since $R(T)$ is closed, by Theorem [4, page 287, V.5], $R\left(T_{i}\right)$ is closed for $i=1,2$. Let $S=\left(\begin{array}{cc}T_{1}^{\dagger} & 0 \\ 0 & T_{2}^{\dagger}\end{array}\right)$. Note that $S \in \mathcal{B}(H)$ and it can be verified that $S$ satisfies all the conditions of the Moore-Penrose inverse. Since $T^{\dagger}$ is unique, it follows that $S=T^{\dagger}$. This proves the claim.

Theorem 4.6. Let $T \in \mathcal{C}(H)$ be densely defined and have a bounded inverse. Let $M$ be a subspace of $H$. Then

(1) $m\left(\left.T\right|_{M}\right)=\frac{1}{\left\|\left.T^{-1}\right|_{T(M \cap D(T))}\right\|}$

(2) if $M$ is closed, then $T(M \cap D(T))$ is closed

(3) If $N$ is any subspace of $H$, then $m\left(\left.T\right|_{T^{-1}(N)}\right)=\frac{1}{\left\|\left.T^{-1}\right|_{N}\right\|}$.

Furthermore, if $N$ is closed and $T \in \mathcal{B}(H)$, then $T^{-1}(N)$ is closed.

(4) $T \in \mathcal{A M}_{c}(H)$ if and only if $T^{-1} \in \mathcal{A N}(H)$. 
Proof. Proof of (1): First, note that as $T$ is one-to-one, we have $D(T)=C(T)$ and $m(T)=\gamma(T)$. By definition,

$$
\begin{aligned}
m\left(\left.T\right|_{M}\right) & =\inf \left\{\frac{\|T x\|}{\|x\|}: x \in M \cap D(T), x \neq 0\right\} \\
& =\frac{1}{\sup \left\{\frac{\|x\|}{\|T x\|}: x \in M \cap C(T), x \neq 0\right\}} \\
& =\frac{1}{\sup \left\{\frac{\left\|T^{-1} y\right\|}{\|y\|}: y=T x \in T(M \cap D(T)), x \neq 0\right\}} \\
& =\frac{1}{\left\|\left.T^{-1}\right|_{T(M \cap D(T))}\right\|} .
\end{aligned}
$$

Proof of (2): Let $N:=T(M \cap D(T))$ and let $y \in \bar{N}$. Let $\left(x_{n}\right) \subset M \cap D(T)$ be such that $y=\lim _{n \rightarrow \infty} T x_{n}$. Since $T^{-1} \in \mathcal{B}(H)$, it follows that $\lim _{n \rightarrow \infty} x_{n}=T^{-1} y$. Since $M$ is closed, we can conclude that $T^{-1} y \in M \cap D(T)$. Since $R(T)$ is closed, $y \in R(T)$. Hence $y=T\left(T^{-1} y\right) \in T(M \cap D(T))$.

Proof of (3): This goes along the similar lines of (1) and (2).

Proof of (4): If $M=H$, then by Theorem 3.20, we have that $T \in \mathcal{M}_{c}(H)$ if and only if $T^{-1} \in \mathcal{N}(H)$. Hence assume that $\{0\} \neq M \subset H$. Let $T^{-1} \in \mathcal{A N}(H)$. Let $X=T(M \cap D(T))$ and $R_{X}=\left.T^{-1}\right|_{X}$. By (2), $X$ is closed. Since $R_{X} \in \mathcal{N}(X, H)$, there exists $y_{0} \in S_{X}$, such that $\left\|R_{X} y_{0}\right\|=\left\|R_{X}\right\|$. This is equivalent to the fact that $R_{X}^{*} R_{X} y_{0}=\left\|R_{X}\right\|^{2} y_{0}$. Let $y_{0}=T x_{0}$ for some $x_{0} \in M \cap D(T)$, we get $R_{X}^{*} x_{0}=\left\|R_{X}\right\|^{2} T x_{0}$. Therefore $\left\|T x_{0}\right\|=\frac{\left\|R_{X}^{*} x_{0}\right\|}{\left\|R_{X}\right\|^{2}} \leq \frac{\left\|x_{0}\right\|}{\left\|R_{X}\right\|}=m\left(\left.T\right|_{M}\right)\left\|x_{0}\right\|$ by (1). Writing $z_{0}=\frac{x_{0}}{\left\|x_{0}\right\|}$, we get that $\left\|T z_{0}\right\| \leq m\left(\left.T\right|_{M}\right)$. But the other inequality holds clearly. Hence $\left.T\right|_{M} \in \mathcal{M}_{c}(M, H)$.

Conversely, assume that $T \in \mathcal{A M}_{c}(H)$. Let $N$ be a closed subspace of $H$ and let $M:=T^{-1}(N) \subseteq C(T)$. Since $T \in \mathcal{A M}_{c}(H)$, we have $T_{M}:=\left.T\right|_{M} \in$ $\mathcal{M}_{c}(M, H)$. It can be easily verified that $T_{M}$ is closed, since $T$ is closed. Since $D(T) \cap M$ is dense in $\overline{D(T) \cap M}, T_{M}$ is densely defined operator. Hence $T_{M}^{*}$ : $D\left(T_{M}^{*}\right) \rightarrow \bar{M}$ exists. By Theorem 3.13 and Proposition 3.11, there exists $x_{0} \in$ $S_{D\left(T_{M}^{*} T_{M}\right)}$ such that

$$
T_{M}^{*} T_{M} x_{0}=m\left(T_{M}\right)^{2} x_{0} .
$$

As $T$ is bounded below, $T_{M}$ is bounded below and hence $m\left(T_{M}\right)>0$. Let $x_{0}=T^{-1} y_{0}$ for some $y_{0} \in N$. Then Equation 4.1 takes the form:

$$
T_{M}^{*} y_{0}=m\left(T_{M}\right)^{2} T^{-1} y_{0} .
$$

First, observe that $R\left(T_{M}\right)=T_{M}(M \cap D(T))=T(D(T) \cap M)=T(M)=$ $T\left(T^{-1}(N)\right)=N$. Hence $y_{0} \in N=R\left(T_{M}\right)=N\left(\left(T_{M}\right)^{*}\right)^{\perp}$. Taking norm both sides of Equation 4.2, we get

$$
\left\|T^{-1} y_{0}\right\|=\frac{\left\|T_{M}^{*} y_{0}\right\|}{m\left(T_{M}\right)^{2}} \geq \frac{\gamma\left(T_{M}^{*}\right)\left\|y_{0}\right\|}{m\left(T_{M}\right)^{2}}=\frac{\gamma\left(T_{M}\right)\left\|y_{0}\right\|}{m\left(T_{M}\right)^{2}} \geq \frac{m\left(T_{M}\right)\left\|y_{0}\right\|}{m\left(T_{M}\right)^{2}}=\frac{\left\|y_{0}\right\|}{m\left(T_{M}\right)} .
$$


Hence $z_{0}=\frac{y_{0}}{\left\|y_{0}\right\|} \in S_{N}$ and $\left\|T^{-1}\left(z_{0}\right)\right\|=\left\|\left.T^{-1}\right|_{N}\right\|$.

Corollary 4.7. Let $T \in \mathcal{B}(H)$ be such that $T^{-1} \in \mathcal{B}(H)$. Then $T \in \mathcal{A M}_{c}(H)$ if and only if $T^{-1} \in \mathcal{A N}(H)$.

Theorem 4.8. Let $T \in \mathcal{A M}_{c}(H)$, positive and not bounded. Assume that $T$ is one-to-one. Then there exists an unbounded (increasing) sequence $\left\{\lambda_{n}\right\}$ of eigenvalues of $T$ with corresponding eigenvectors $\left\{\phi_{n}\right\}$ such that

$$
\begin{gathered}
D(T)=\left\{x \in H: \sum_{n=1}^{\infty} \lambda_{n}^{2}\left|\left\langle x, \phi_{n}\right\rangle\right|^{2}<\infty\right\} \text { and } \\
T x=\sum_{n=1}^{\infty} \lambda_{n}\left\langle x, \phi_{n}\right\rangle \phi_{n}, \text { for all } x \in D(T) .
\end{gathered}
$$

The series in the above representation converges in the strong operator topology. Moreover, $T^{-1}$ is compact.

(2) $\sigma(T)=\left\{\lambda_{n}: n \in \mathbb{N}\right\}=\sigma_{p}(T)$

(3) if $\mu \in \sigma_{p}(T)$, then $\mu$ is an eigenvalue with finite multiplicity

(4) $\overline{\operatorname{span}}\left\{\phi_{n}: n \in \mathbb{N}\right\}=H$.

Proof. Proof of (1): First note that as $T$ is one-to-one and $R(T)$ is closed, $T$ is bounded below. Since $T \geq 0, T^{-1}$ exists and bounded. If $T \in \mathcal{A M}_{c}(H)$, then $T^{-1} \in \mathcal{A N}(H)$ by Theorem 4.6. Hence by [3, Theorem 2.5], there exists unique triple $(K, F, \alpha)$, where $K \in \mathcal{K}(H)$ is positive, $F \in \mathcal{F}(H)$ positive and $\alpha \geq 0$ such that $K F=0=F K, F \leq \alpha I$ and $T^{-1}=\alpha I+K-F$. If $\alpha=0$, then $F=0$ and hence $T^{-1}=K \in \mathcal{K}(H)$. Next, assume that $\alpha>0$. In this case, $R\left(T^{-1}\right)=D(T)$ is closed by [3, Proposition 2.8] is closed. Since $T$ is densely defined, we must have that $D(T)=H$. By the closed graph theorem $T$ must be bounded, a contradiction. Hence $\alpha>0$ is not possible. This implies that $\alpha=0$ and hence $T^{-1} \in \mathcal{K}(H)$.

By the spectral theorem, there exists increasing sequence $\left(\mu_{n}\right)$ of positive eigenvalues of $T^{-1}$ with corresponding eigenvectors $\left\{\phi_{n}: n \in \mathbb{N}\right\}$ such that

$$
T^{-1} y=\sum_{n=1}^{\infty} \mu_{n}\left\langle y, \phi_{n}\right\rangle \phi_{n}, \text { for all } y \in H \text {. }
$$

The sequence $\mu_{n} \rightarrow 0$ as $n \rightarrow \infty$. More over, the above series converges to $T^{-1}$ in the operator norm of $\mathcal{B}(H)$. We can also observe that the sequence $\left(\mu_{n}\right)$ is an infinite sequence. Otherwise, $T^{-1}$ is a finite rank operator and $\sigma(T)$ is bounded. By [26] this implies that $T$ is bounded which leads to a contradiction. Also, since $T^{-1}$ is compact, $\sigma\left(T^{-1}\right)=\left\{\mu_{n}: n \in \mathbb{N}\right\}=\sigma_{p}(T)$ and each $\mu_{n}$ has finite multiplicity. Also, $\mu_{n+1} \leq \mu_{n}$ for each $n \in \mathbb{N}$. 
Let $\lambda_{n}:=\mu_{n}^{-1}$ for all $n \in \mathbb{N}$. As $T^{-1}$ is compact, by [10, Theorem 6.1, page 214], it follows that

$$
\begin{gathered}
D(T)=\left\{x \in H: \sum_{n=1}^{\infty} \lambda_{n}^{2}\left|\left\langle x, \phi_{n}\right\rangle\right|^{2}<\infty\right\} \text { and } \\
T x=\sum_{n=1}^{\infty} \lambda_{n}\left\langle x, \phi_{n}\right\rangle \phi_{n}, \text { for all } x \in D(T) .
\end{gathered}
$$

On the other hand, if $T^{-1}$ is compact, by Theorem 4.6, $T \in \mathcal{A M}_{c}(H)$.

Proof of statement (2) is clear. The statement (3) is proved in (1).

Proof of (4): Since $T^{-1}$ is compact, $R\left(T^{-1}\right)=D(T)$ is separable and by the representation above, we have that $H=\overline{D(T)}=\overline{R\left(T^{-1}\right)}=\overline{\operatorname{span}}\left\{v_{n}: n \in \mathbb{N}\right\}$.

Remark 4.9. If $T \in \mathcal{B}(H)$, then the conclusion (1) of Theorem 4.8 is not true. The unboundedness of the operator is used to get the inverse to be compact.

Theorem 4.10. Let $T \in \mathcal{C}(H)$ be densely defined and one-to-one but not bounded. Then

(1) $T \in \mathcal{A M}_{c}(H)$ if and only if $T^{*} T \in \mathcal{A M}_{c}(H)$

(2) $T \in \mathcal{A M}_{c}(H)$ if and only if $T^{\dagger} \in \mathcal{K}(H)$.

Proof. Proof of (1): If $T \in \mathcal{A M}_{c}(H)$, then $R(T)$ is closed. As $T$ is one-to-one, $T$ is bounded below. Also, since $|T|$ and $T^{*} T$ are bounded below and positive, both have bounded inverse. Hence

$$
\begin{aligned}
T \in \mathcal{A M}_{c}(H) \Leftrightarrow|T| \in \mathcal{A} \mathcal{M}_{c}(H) & \Leftrightarrow|T|^{-1} \in \mathcal{K}(H) \text { (by Theorem 4.8) } \\
& \Leftrightarrow|T|^{-2} \in \mathcal{K}(H) \\
& \Leftrightarrow\left(T^{*} T\right)^{-1} \in \mathcal{K}(H) \\
& \Leftrightarrow T^{*} T \in \mathcal{A M}_{c}(H) \text { (by Theorem 4.8). }
\end{aligned}
$$

Proof of (2): By (1),

$$
\begin{aligned}
T \in \mathcal{A M}_{c}(H) & \Leftrightarrow|T| \in \mathcal{A} \mathcal{M}_{c}(H) \quad \Leftrightarrow|T|^{-1} \in \mathcal{K}(H) \text { (by Theorem 4.8) } \\
& \Rightarrow|T|^{\dagger} \in \mathcal{K}(H) .
\end{aligned}
$$

On the other hand, if $T^{\dagger} \in \mathcal{K}(H)$, then $R(T)$ is closed. As $T$ is one-to-one, $T$ must be bounded below. This implies that $|T|^{-1} \in \mathcal{B}(H)$. Thus,

$$
\begin{aligned}
T^{\dagger} \in \mathcal{K}(H) \Leftrightarrow\left(T^{*}\right)^{\dagger} \in \mathcal{K}(H) & \Leftrightarrow\left|\left(T^{*}\right)^{\dagger}\right| \in \mathcal{K}(H) \\
& \Leftrightarrow|T|^{\dagger}=|T|^{-1} \in \mathcal{K}(H) \text { (by (2) of Proposition 3.19) } \\
& \Leftrightarrow|T| \in \mathcal{A M}_{c}(H) \\
& \Leftrightarrow T \in \mathcal{A M}_{c}(H) .
\end{aligned}
$$

Theorem 4.11. Let $T \in \mathcal{C}(H)$ be densely defined unbounded and have a bounded inverse. Then $T \in \mathcal{A M}_{c}(H)$ if and only if $T^{*} \in \mathcal{A M}_{c}(H)$. 
Proof. First observe that both $T$ and $T^{*}$ are bounded below. We know that $T \in$ $\mathcal{A M}_{c}(H)$ if and only if $T^{-1} \in \mathcal{K}(H)$. This is true if and only if $\left(T^{*}\right)^{-1} \in \mathcal{K}(H)$. Now, by 2 of Theorem 4.10, this is equivalent to the fact that $T^{*} \in \mathcal{A M}_{c}(H)$.

Definition 4.12 (Hyper invariant subspace). Let $T \in \mathcal{C}(H)$ be densely defined and $M$ be a closed subspace of $H$. Then $M$ is said to be hyperinvariant subspace of $T$ if $M$ is invariant under every $S \in \mathcal{B}(H)$ such that $S T \subseteq T S$.

Theorem 4.13. (Lomonosov)[22] Every operator that commutes with a non-zero compact operator and is not a multiple of the identity has a non-trivial hyperinvariant subspace.

Using Theorem 4.13 we will prove that every $\mathcal{A} \mathcal{M}$-operator has a non trivial hyperinvariant subspace.

Theorem 4.14. Let $T \in \mathcal{A M}_{c}(H)$, unbounded and $T^{-1} \in \mathcal{B}(H)$. Then $T$ has a non trivial hyper invariant subspace.

Proof. Let $S \in \mathcal{B}(H)$ be such that $S T \subseteq T S$. That is $S T x=T S x$ for all $x \in D(T)$. Then it can be easily verified that $T^{-1} S=S T^{-1}$. But $T^{-1} \in \mathcal{K}(H)$ by (2) of Theorem 4.10. Now, by Theorem 4.13, $T^{-1}$ has a non trivial invariant subspace, say $M$. Then $M$ is invariant under $S$. Thus the conclusion follows.

Now, we can drop the condition that the operator to be one-to-one in Theorem 4.8 and prove the result.

Theorem 4.15. Let $T \in \mathcal{A M}_{c}(H)$ be, positive but not bounded. Then

(1) $T^{\dagger}$ is compact

(2) $R(T)$ is separable.

Proof. Since $N(T)$ reduces $T$, we can write $T=\left(\begin{array}{cc}T_{0} & 0 \\ 0 & T_{1}\end{array}\right)$, where $T_{0}=\left.T\right|_{N(T)}$ and $T_{1}=T_{N(T)^{\perp}}$. Then by Lemma $4.5, T^{\dagger}=\left(\begin{array}{cc}T_{0}^{\dagger} & 0 \\ 0 & T_{1}^{-1}\end{array}\right)$. As $T_{1} \in \mathcal{A M}\left(N(T)^{\perp}\right)$, by Theorem 4.10, $T_{1}^{-1}$ is compact. Note that $T_{0}=0$ if $N(T) \neq\{0\}$ and $T=T_{1}$ if $N(T)=\{0\}$. Hence $T^{\dagger}$ is compact. Also $R\left(T_{1}\right)$ is separable by (4) of Theorem 4.8. Now the conclusion follows as $R(T)=R\left(T_{1}\right)$.

Using Theorem 4.15, we can prove a more general result.

Theorem 4.16. Let $T \in \mathcal{A M}_{c}(H)$, but not bounded. Then

(1) $T^{\dagger}$ is compact

(2) $N(T)^{\perp}$ and $R(T)$ are separable.

Proof. Proof of (1): We have $T \in \mathcal{A M}_{c}(H)$ if and only if $|T| \in \mathcal{A M}_{c}(H)$. Hence $|T|^{\dagger} \in \mathcal{K}(H)$ by Theorem 4.15. But, by Proposition 3.19, $|T|^{\dagger}=\left|\left(T^{\dagger}\right)^{*}\right|$ and hence $\left(T^{\dagger}\right)^{*} \in \mathcal{K}(H)$. This implies that $T^{\dagger} \in \mathcal{K}(H)$.

Proof of (2): Since $T^{\dagger}$ is compact, $R\left(T^{\dagger}\right)=C(T)$ is separable. Hence $N(T)^{\perp}$ is separable. Since $R(T)$ is closed, $R\left(T^{*}\right)$ must be closed and since $N(T)^{\perp}=R\left(T^{*}\right)$ , $R\left(T^{*}\right)$ is separable. But, $R\left(T^{*}\right)$ is separable if and only if $R(T)$ is separable by [23, Problem 11.4.6, page 362]. 
Question 4.17. If $T \in \mathcal{C}(H)$ is densely defined and $T^{\dagger} \in \mathcal{K}(H)$. Is it true that $T \in \mathcal{A M}_{c}(H)$.

Theorem 4.18. Let $T$ be a densely defined and self-adjoint, one-to-one operator on an infinite dimensional Hilbert space $H$ which is not bounded. Then the following are equivalent:

(1) $T \in \mathcal{A M}_{c}(H)$

(2) $T^{-1} \in \mathcal{K}(H)$

(3) there exists a real sequence $\left(\lambda_{n}\right)$ and an orthonormal basis $\left\{v_{n}: n \in \mathbb{N}\right\}$ of $H$ such that $\lim _{n \rightarrow \infty}\left|\lambda_{n}\right|=\infty$ and $T v_{n}=\lambda_{n} v_{n}$ for each $n \in \mathbb{N}$

(4) $T$ has purely discrete spectrum

(5) the resolvent $R_{\lambda}(T):=(T-\lambda I)^{-1}$ is compact for one, and hence for all $\lambda \in \rho(T)$

(6) the embedding map $J_{T}:\left(D(T),\|\cdot\|_{T}\right) \rightarrow H$ is compact (here $\|x\|_{T}=$ $\left.\left(\|x\|^{2}+\|T x\|^{2}\right)^{\frac{1}{2}}, x \in D(T)\right)$.

Proof. If $T \in \mathcal{A M}_{c}(H)$, then $R(T)$ is closed. As $T$ is one-to-one, $T$ must be bounded below and hence $T^{-1} \in \mathcal{B}(H)$. Now, by (2) of Theorem 4.10, $T^{-1} \in$ $\mathcal{K}(H)$. Again by (2) of Theorem 4.10, if $T^{-1} \in \mathcal{K}(H)$, then $T \in \mathcal{A M}_{c}(H)$. Thus (1) and (2) are equivalent. The equivalence of (2)-(6) follows by [27, Proposition 5.12 , page 94].

Next, we give an example of $\mathcal{A} \mathcal{M}$-operator.

Example 4.19. Let $p, p^{\prime}, q, w$ be continuous real valued functions defined on $[a, b]$ with $a<b$ and $w(t)>0$ for all $t \in[a, b]$. Consider the real Hilbert space

$$
H:=\left\{u: \int_{a}^{b}|u|^{2} w<\infty\right\}
$$

with the inner product

$$
\langle u, v\rangle:=\int_{a}^{b} u(x) v(x) w(x) d x .
$$

Let $L$ be the Sturm-Liouville operator given by

$$
L u:=\frac{1}{w}\left[-\left(p u^{\prime}\right)^{\prime}+q u\right]
$$

with

$$
D(L)=\left\{\begin{aligned}
\beta_{1} u(a)+\gamma_{1} u^{\prime}(a) & =0, \\
\beta_{2} u(b)+\gamma_{2} u^{\prime}(b) & =0, \\
\left|\beta_{1}\right|+\left|\gamma_{1}\right| & >0, \\
\left|\beta_{2}\right|+\left|\gamma_{2}\right| & >0
\end{aligned}\right\} .
$$

Since $D(L)$ contains continuous functions defined on $[a, b]$ with compact support, $L$ is densely defined operator. Also $L$ is symmetric (See [9, Chapter 7, section 5]. Let us assume that $0 \notin \sigma_{p}(L)$. In this case it easy to see that $L^{-1}$ is compact and self adjoint. Let $B:=\left\{\phi_{1}, \phi_{2}, \ldots\right\}$ is an orthonormal basis for $H$ such that 
$L v_{n}=\mu_{n} v_{n}$, where $\mu_{1}, \mu_{2}, \ldots$ is a sequence of real numbers which are eigenvalues of $L$. In this case every $u \in H$ can be expressed as

$$
u=\sum_{n=1}^{\infty}\left\langle u, v_{n}\right\rangle v_{n}
$$

If $u \in D(L)$, then

and

$$
\sum_{n=1}^{\infty}\left|\left\langle u, v_{n}\right\rangle\right|^{2} \mu_{n}^{2}<\infty
$$

$$
L u=\sum_{n=1}^{\infty} \mu_{n}\left\langle u, v_{n}\right\rangle v_{n}
$$

Note that $L^{-1} y=\sum_{n=1}^{\infty} \mu_{n}^{-1}\left\langle y, v_{n}\right\rangle v_{n}$ for all $y \in H$. It is clear that $\left|L^{-1}\right|$ is compact and by Proposition 3.19, we have $|L|^{-1}=\left|L^{-1}\right|$. Hence by Theorem 4.18, $L \in \mathcal{A M}_{c}(H)$.

We end up this section with the following question:

Question 4.20. Does every bounded absolutely minimum attaining operator have a non trivial (hyper) invariant subspace?

Author contributions All authors contributed equally and significantly in this paper. All authors read and approved the final manuscript.

\section{Compliance with ethical standards}

Conflict of interest The authors declare that they have no conflict of interest. Ethical approval: This article does not contain any studies with human participants or animals performed by any of the authors.

\section{REFERENCES}

[1] N. I. Akhiezer and I. M. Glazman, Theory of linear operators in Hilbert space, translated from the Russian and with a preface by Merlynd Nestell, reprint of the 1961 and 1963 translations, Dover Publications, Inc., New York, 1993. MR1255973

[2] M. D. Acosta, R. M. Aron and F. J. García-Pacheco, The approximate hyperplane series property and related properties, Banach J. Math. Anal. 11 (2017), no. 2, 295-310. MR3598746

[3] D. Venku Naidu and G. Ramesh, On absolutely norm attaining operators, to appear in Proc. Indian Acad. Sci. Math. Sci. (https://www.ias.ac.in/public/Volumes/pmsc/forthcoming/PMSC-D-18-00008.pdf)

[4] A. E. Taylor and D. C. Lay, Introduction to functional analysis, second edition, John Wiley \& Sons, New York, 1980. MR0564653 (81b:46001)

[5] S. Goldberg, Unbounded linear operators: Theory and applications, McGraw-Hill, New York, 1966. MR0200692 (34 \#580)

[6] A. Ben-Israel and T. N. E. Greville, Generalized inverses: theory and applications, WileyInterscience, New York, 1974. MR0396607 (53 \#469)

[7] W. Rudin, Functional analysis, second edition, International Series in Pure and Applied Mathematics, McGraw-Hill, New York, 1991. MR1157815 (92k:46001)

[8] T. Kato, Perturbation theory for linear operators, second edition, Springer, Berlin, 1976. MR0407617 (53 \#11389) 
[9] A. W. Naylor and G. R. Sell, Linear operator theory in engineering and science, second edition, Applied Mathematical Sciences, 40, Springer, New York, 1982. MR0672108 (83j:46001)

[10] I. Gohberg, S. Goldberg and M. A. Kaashoek, Basic classes of linear operators, Birkhäuser, Basel, 2003. MR2015498 (2005g:47001)

[11] Bernau, S. J. Extreme eigenvectors of a normal operator. Proc. Amer. Math. Soc. 181967 127-128. MR0205066 (34 \#4901)

[12] Carvajal, Xavier; Neves, Wladimir. Operators that achieve the norm. Integral Equations Operator Theory 72 (2012), no. 2, 179-195. MR2872473 (2012k:47044)

[13] X. Carvajal and W. Neves, Operators that attain their minima, Bull. Braz. Math. Soc. (N.S.) 45 (2014), no. 2, 293-312. MR3249529

[14] J. Falcó et al., Spaceability in norm-attaining sets, Banach J. Math. Anal. 11 (2017), no. 1, 90-107. MR3571146

[15] J. Ganesh, G. Ramesh and D. Sukumar, Perturbation of minimum attaining operators, Adv. Oper. Theory 3 (2018), no. 3, 473-490. MR3795095

[16] J. Ganesh, G. Ramesh and D. Sukumar, A characterization of absolutely minimum attaining operators, J. Math. Anal. Appl. 468 (2018), no. 1, 567-583. MR3849004

[17] G. Ramesh, Approximation methods for solving operator equations involving unbounded operators, Ph. D Thesis, IIT Madras. (2008).

[18] P. Enflo, J. Kover and L. Smithies, Denseness for norm attaining operator-valued functions, Linear Algebra Appl. 338 (2001), 139-144. MR1861118 (2002g:47148)

[19] S. Shkarin, Norm attaining operators and pseudospectrum, Integral Equations Operator Theory 64 (2009), no. 1, 115-136. MR2501174 (2010c:47004)

[20] M. Š. Birman and M. Z. Solomjak, Spectral theory of selfadjoint operators in Hilbert space (Russian), Leningrad. Univ., Leningrad, 1980. MR0609148 (82k:47001)

[21] G. Ramesh, Structure theorem for $\mathcal{A N}$-operators, J. Aust. Math. Soc. 96 (2014), no. 3, 386-395. MR3217722

[22] H. Radjavi and P. Rosenthal, Invariant subspaces, second edition, Dover, Mineola, NY, 2003. MR2003221 (2004e:47010)

[23] Y. A. Abramovich and C. D. Aliprantis, An invitation to operator theory, Graduate Studies in Mathematics, 50, Amer. Math. Soc., Providence, RI, 2002. MR1921782 (2003h:47072)

[24] S. H. Kulkarni and G. Ramesh, Projection methods for computing Moore-Penrose inverses of unbounded operators, Indian J. Pure Appl. Math. 41 (2010), no. 5, 647-662. MR2735209 (2012k:47001)

[25] S. H. Kulkarni and G. Ramesh, On the denseness of minimum attaining operators, Oper. Matrices 12 (2018), no. 3, 699-709. MR3853362

[26] S. H. Kulkarni and M. T. Nair, A characterization of closed range operators, Indian J. Pure Appl. Math. 31 (2000), no. 4, 353-361. MR1760936 (2001d:47009)

[27] Schmüdgen, Konrad, Unbounded self-adjoint operators on Hilbert space, Graduate Texts in Mathematics, 265, Springer, Dordrecht, 2012, xx+432, MR2953553

[28] S. K. Pandey and V. I. Paulsen, A spectral characterization of $\mathcal{A N}$ operators, J. Aust. Math. Soc. 102 (2017), no. 3, 369-391. MR3650963

${ }^{1}$ Department of Mathematics, I. I. T. Madras, Chennai, Tamilnadu, India 600 036.

E-mail address: shk@iitm.ac.in

${ }^{2}$ Department of Mathematics, I. I. T. Hyderabad, Kandi(V), Sangareddy, TelanGANA, INDIA-502 285.

E-mail address: rameshg@iith.ac.in 